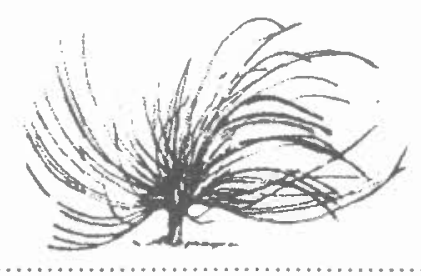

\title{
La investigación dirigida como un método alternativo en la enseñanza de las ciencias'
}

\author{
Alejandro Moya Segura ${ }^{2}$ \\ Universidad Nacional, Costa Rica \\ Heredia, Costa Rica \\ ensayandoenlauna@gmail.com
}

Esteban Chaves Sibaja

Universidad Nacional, Costa Rica

Heredia, Costa Rica

echfisica@gmail.com

\section{Kenneth Castillo Rodriguez ${ }^{4}$ Universidad Nacional, Costa Rica \\ Heredia, Costa Rica kencasrod@gmail.com}

\begin{abstract}
Resumen
En el presente artículo se pone en perspectiva y se analiza detalladamente una de las estrategias de aprendizaje dirigida a la enseñanza de las Ciencias y denominada como: investigación dirigida. Se pone en perspectiva el origen histórico de esta estrategia junto con otras, respondiendo
\end{abstract}

Recibido: 08 de setiembre, 2010 - Aprobado: 29 de junio, 2011

1 El presente artículo es el resultado de un proceso continuo de discusión y análisis llevado a cabo en el curso de Seminario de Debates Pedagógicos correspondiente a la licenciatura en la Enseñanza de las Ciencias Naturales de la Universidad Nacional, Heredia (2010), con el propósito de contribuir en el mejoramiento y la calidad de la educación científica en Costa Rica.

2 Bachiller en Enseñanza de las Ciencias de la Universidad Nacional. Estudiante de la licenciatura de la Cartera de Enseñanza de las Ciencias Naturales de la Universidad Nacional.

3 Bachiller en Enseñanza de las Ciencias de la Universidad Nacional. Estudiante de la de la licenciatura de la Carrera de Enseñanza de las Ciencias Naturales de la Universidad Nacional.

4 Bachiller en Enseñanza de las Ciencias de la Universidad Nacional. Estudiante de la licenciatura de la Carrera de Enseñanza de las Ciencias Naturales de la Universidad Nacional. 
así a un cambio obligado de la educación bancaria y asociacionista, un modelo educativo que toma en cuenta al estudiante, a su ambiente y respondiendo a su inquietudes, detalla además una serie de aspectos a considerar para utilizarlo en las clases de Ciencias de secundaria de Costa Rica, así como a complementarla con otras estrategias con tendencias constructivistas y a las muy recientes estrategias metacognitivas, para garantizar un aprendizaje significativo de los temas tratados y respetando la diversidad de estilos de aprendizaje y las inteligencias múltiples, se menciona además los posibles alcances y limitaciones de la estrategia aplicada en el aula; la idea general de la enseñanza mediante la investigación dirigida es que el estudiante construya por sí mismo y mediante el método científico un cuerpo de conocimientos que le permita explicar científicamente fenómenos cotidianos que son intrigantes para él, eso sí, todo el tiempo guiado por el docente .

Palabras claves: Enseñanza de las Ciencias, Investigación Dirigida, Aprendizaje Significativo, aprender a aprender, constructivismo, Estrategias de Aprendizaje, Aprendizaje por Descubrimiento, Conflicto Cognitivo, humanismo, Metacognición, Educación Bancaria, Proceso Educativo.

\begin{abstract}
This paper presents and analyzes a learning strategy devoted to the teaching of Science which is called: guided research. It shows the historical origin of this strategy promoting a mandatory change in the banking and associationist teaching, a teaching model which takes into account the student and his teaching environment. It also explains a series of aspects to be used in the high school science classes in Costa Rica; as well as other metacognitive and constructivist trends in order to ensure a meaningful learning on the studied topic considering the different learning styles and multiple intelligences. It also mentions the possible scope and limitations of the applied strategy. The main idea of using the guided research is that by using the scientific method, students built by themselves a body of knowledge that allows them to scientifically explain intriguing daily phenomena.
\end{abstract}


Keywords: Science teaching, guided research, meaningful learning, learning to learn, constructivism, learning strategies, discovery learning, cognitive conflict, humanism, metacognition, banking teaching, teaching process.

\title{
Introducción
}

\begin{abstract}
A partir de la década de los años setenta hasta la fecha, se ha venido gestando un cambio en la manera que se enseña, ya que para entonces el arraigado sistema de enseñanza basado en lo que Freire llamó "educación bancaria", no estaba dando los frutos que los mismos profesores esperaban.
\end{abstract}

Surge entonces, en el caso de la enseñanza de las Ciencias, una estrategia de aprendizaje, denominada "aprendizaje por descubrimiento", cuya esencia recaía en que los estudiantes aprendieran Ciencia, haciendo Ciencia, tal y como trabajan los científicos. El espiritu de tal estrategia no era malo, el gran problema surgió porque los docentes satanizaron la enseñanza por descubrimiento, y pensaban que a las y los alumnos había que dejarlos solos a la hora en que hacían Ciencia, sin ninguna mediación. Claro está que esta estrategia tal y como se concibió no tuvo mucho éxito y con el pasar de los años fue adquiriendo muchos detractores que abogaban por su modificación.

El proceso educativo tiene como fin último formar ciudadanos y ciudadanas, con las habilidades y destrezas esenciales para que puedan aprender por sí solos durante toda su vida, y no sólo durante su periodo escolar (normalmente desde primaria hasta la educación superior)

Por ello, el docente debe enseñar a través de metodologías, que no sólo le permitan al estudiante adquirir y asimilar conceptos de una determinada disciplina, sino también que esas metodologías permitan que esa persona en formación adquiera conocimientos, para poder diseñar él sus propias metodologías con el fin último de resolver las situaciones problematizadoras que surgen en nuestros hogares, en los centros de trabajo e incluso en las actividades recreativas.

La Ciencia, por el tipo de conceptos que maneja, le permite a las personas (que no necesariamente son científicos o científicas) conocer e interpretar su realidad. Y no sólo sus conceptos: también las estrategias para el aprendizaje de tales conceptos, pueden extrapolarse al aprendizaje de la vida; al resolver los problemas que esta presenta. 
Bajo esta premisa se está pasando de una Ciencia positivista a una Ciencia al servicio del humanismo, que no sólo hace crecer a las personas en conocimientos sino también en criticidad, pudiendo tomar decisiones objetivas sobre cómo enfrentar las diferentes situaciones que la vida nos pone día a día.

\section{Nuevas tendencias en la enseñanza de las ciencias}

La práctica educativa es una de las profesiones más activas que existen. Constantemente se debe de ir renovando la manera en que se enseña, ya que cada día hay nuevos avances al descifrar la manera en que las personas aprendemos. Desde el punto de vista fisiológico y psicológico, las nuevas generaciones tienen nuevos intereses, así que el conocimiento hay que adaptarlo a sus gustos para que adquiera algún significado en los alumnos, y en el caso de la enseñanza de las Ciencias, los avances científicos se dan constantemente, así que lo que hoy es verdad mañana puede no serlo, lo que exige por parte del profesorado la actualización y renovación constante.

La enseñanza de las Ciencias, según Núñez (2000) a pesar de la influencia de diferentes corrientes que proponen la utilización de múltiples formas de enseñar las ciencias, es en la que se incentiva la realización de experimentos y demostraciones en la clase, observaciones en el campo con la participación activa de los estudiantes en el proceso de enseñanza y aprendizaje, con el fin de construir los conocimientos en forma conjunta, contextualizados y ligados a la actividad diaria de las personas.

La formación profesional de los docentes de Ciencias, se informa más que formar sobre una serie de métodos alternativos que se pueden utilizar para enseñar Física, Química o Biología, sin recurrir en técnicas basadas en teorías asociacionistas, donde el estudiante es visualizado como un recipiente en el cual el profesor deposita los conocimientos y favorece de manera única el memorismo, más que la construcción activa del conocimiento.

Sin embargo, durante los numerosos espacios programados en nuestra formación, sólo se tiene la posibilidad de aprender a utilizar algunas de las tantas estrategias metodológicas disponibles para enseñar Ciencias.

Las nuevas estrategias de enseñanza de las Ciencias van orientadas a sufragar las carencias que se tienen en la enseñanza científica: 
Un currículo de ideas inconexas que no adquieren ningún significado para los alumnos y estructurado en función de la misma ciencia. De manera que los estudiantes piensan que aprender ciencias es nada más memorizar ecuaciones y definiciones, más que entenderlas y aplicarlas a su realidad. (Driver, 1986; Pozo y Carretero, 1987; Gil, Furió, Valdés, Salinas, et al, 1999)

Estas nuevas estrategias toman en cuenta los conocimientos previos de los estudiantes que, en el caso de las Ciencias, se generan a partir del uso de términos científicos en el lenguaje común y la influencia del entorno.(Moreira y Greca, 2003 )

Las recientes tendencias en enseñanza de las Ciencias, son estrategias basadas en que los y las estudiantes construyan sus conocimientos, a partir de sus concepciones previas, mediante un aprendizaje significativo.

Un aprendizaje significativo de conocimientos científicos requiere la participación de los estudiantes en la constante reconstrucción de los conocimientos que habitualmente se transmiten ya elaborados. (Driver, 1986)

Hoy no se habla de métodos de enseñanza debido a que enseñar es una situación problematizadora muy abierta, por lo que se ha migrado al término de estrategias de aprendizaje, sistematizadas en actividades de enseñanza muchas veces asociados a métodos didácticos concretos. (Campanario y Moya, 1999)

Bajo toda esta fundamentación han surgido estrategias como: la enseñanza por descubrimiento, la enseñanza expositiva, el conflicto cognitivo, la resolución de problemas y la investigación dirigida que es de lo que trata el presente ensayo y la muy poco desarrollada metacognición (Pozo y Gómez, 1998; Campanario y Moya, 1999).

Mucho más reciente en las nuevas tendencias de enseñanza de las ciencias, es el notable auge que ha tenido la metacognición, cuyo sustento recae en la comprensión de la relevancia de la información o en qué es relevante en el aprendizaje; aún no se tiene muy bien definido cuales son la mejores estrategias para su evaluación sin embargo se trabaja ello. Entre algunas estrategias basadas en la metacognición se tiene el uso de mapas conceptuales, $\mathrm{V}$ heurísticas, resolución de problemas, autocuestionarios y el uso de diarios. (Campanario, 2000).

El ideal de enseñar Ciencias consiste en conseguir que los alumnos sustituyan sus ideas intuitivas, pero muy arraigadas de los fenómenos 
científicos, por otros conceptos más avanzados y más próximos a las teorías científicas admitidas y generar así el cambio conceptual, generar el conflicto cognitivo. (Posner, Strike, Hewson, Gertzog, 1982; Pozo y Carretero, 1987)

Seguidamente se hace un análisis de un método alternativo para la enseñanza de las ciencias según las nuevas tendencias: La Investigación Dirigida. Su espíritu radica en el trabajo en equipo, explicación de la realidad próxima de los y las estudiantes y la contrastación de ideas entre sus semejantes, con el profesor, con científicos o con los libros de texto. Su fundamentación: La construcción activa de su conocimiento, el aprender a aprender.

\section{La Investigación Dirigida}

El presente artículo profundiza en una de las formas de enseñar Ciencias: la Enseñanza mediante la Investigación Dirigida o también llamada Investigación Orientada (Furió y Guisasola, 2001). Conforme se avance en el mismo se tratará de situar este tipo de enseñanza en el sistema educativo costarricense y se expondrán las bases del mismo para su mejor entendimiento.

En la Enseñanza de las Ciencias, casi siempre se hace una clara distinción entre lo que son las prácticas de laboratorio, la enseñanza de conceptos y lo que es la práctica de resolución de ejercicios directamente sobre papel. Debido a esto en los cursos universitarios las materias teóricas y prácticas se dan por separado e inclusive con profesores diferentes; esa diferencia se realiza también a la hora de investigar e innovar con nuevas estrategias didácticas para la enseñanza de las ciencias. (Gil; Furió; Valdés; Salinas; et al., 1999)

Esa tradición en la enseñanza de las ciencias, la hemos asumido acríticamente y no se han realizado muchos esfuerzos con tal de unificar la ciencia. ¿Acaso los científicos distinguen entre un concepto, un número y un fenómeno?. Los tres son componentes esenciales de la Ciencia y con la falta de uno no se puede comprender. (Gil, 1999)

Hoy surge entonces una clara preocupación de los y las docentes por el mejorar su práctica profesional, debido a que han detectado posibles dificultades en el proceso de aprendizaje de sus estudiantes, al usar los métodos tradicionales de enseñanza, ya sea en cualquiera de las tres áreas de la ciencia (Química, Física o Biología), además del uso de 
métodos magistrales de enseñanza, donde el docente imparte los conocimientos a los y las estudiantes de forma pasiva.

Recientes investigaciones en el área de la didáctica de las ciencias, citadas por Gil, et al (1999) apoyan la idea de integrar el aprendizaje de conceptos, la resolución de problemas y el trabajo práctico; tales componentes complementariamente se aprenden mejor cuando los y las estudiantes participan en la investigación científica con el debido apoyo y espacio para la reflexión.

Ya que según Gil (1994) uno de los mayores problemas de la enseñanza de las ciencias es el abismo que existe entre las situaciones de enseñanza-aprendizaje y el modo en que se construye el conocimiento científico

La investigación que detecta problemas (o dudas), se formula y se resuelve, caracterizando el problema como una dificultad que no pueda superarse automáticamente, siendo esta última frase lo que en el proceso de mediación pedagógica lo genere la construcción del conocimiento. (Cañal y Porlan, 1987)

El quehacer de la Ciencia se basa en el método científico. La investigación científica surge de la observación, donde se trata de identificar el fenómeno que causa una inquietud, el científico genera toda una metodología para probar las posibles hipótesis que expliquen tal fenómeno, por último concluye basado en sus resultados experimentales cuál hipótesis responde al fenómeno que le ocasionó la duda, claro está basado siempre en la teoría científica previa y sujeto a variantes que con el tiempo puedan surgir.

Tal proceso genera un aprendizaje. Si la investigación a partir de una inquietud funciona para los científicos, porqué no pensar que para ciertos temas de los programas de estudio de Ciencias en educación secundaria, la investigación podria ser una estrategia metodológica pertinente para el proceso de enseñanza - aprendizaje de tal tema.

Así como menciona Diego-Rasilla (2004) que la utilización de la investigación dentro de un aula implica necesariamente la puesta en práctica del pensamiento científico, es por lo tanto un modo de indagar en la realidad, que no necesariamente está reservado para científicos. Obviamente hoy día se sabe que este no es el único método por el cual generar conocimiento, pero si uno de los más aceptados por las diversas disciplinas del saber. 
Por ejemplo, la investigación en el aula constaría de su marco conceptual, que junto con la ayuda del docente el o la estudiante sería capaz de solucionar los problemas inherentes a los conceptos en que tenga duda o se desconocen y si se tiene la posibilidad, los conceptos se pueden poner en práctica al explicar el fenómeno que generó la interrogante, y por qué no, otros fenómenos cotidianos bajo los cuales se pueden controlar y modificar ciertas variables.

Con esto no sólo se responde la interrogante que generó la investigación en el aula, sino que se le logra dar a la ciencia una aplicabilidad cotidiana - una contextualización - que en la mayor parte de los casos no se logra cuando se fragmenta y se construye el proceso de mediación del conocimiento de forma aislada.

Esta novedosa manera de enfocar el aprendizaje de conceptos científicos, posiciona al estudiante como el eje central de su aprendizaje, pasa a ser sujeto activo, ya que tiene que idear toda una concepción metodológica para lograr responder su interrogante, tiene que retomar y aprender nuevos conceptos (lo que implica un reordenamiento de su estructura cognoscitiva) y por último reorganizar todo ese nuevo conocimiento para generar una respuesta a su incógnita.

Esto no se logra dejando al estudiante investigar sólo, -sin más ni más-. Evidentemente, el docente debe estar presente para que guíe el proceso, diseñe estrategias para acercar los nuevos conocimientos a lo que el estudiante ya conoce, y consecuentemente, debe dar un seguimiento constante al proceso de investigación, de manera que, si este no está funcionando o no va generando una explicación aceptable al problema, sea corregido y retomado (Gil, et al., 1999 y Pozo y Gómez, 1998).

No hay que olvidarse de la educación en valores que genera el realizar una investigación pues, está no es solo generar un conocimiento a partir de una duda, sino también contribuye a la formación del estudiante como un ser social, en valores como disciplina, voluntad, tenacidad y las relaciones interpersonales (Alfonso 2004). Sin dejar de lado los valores inherentes a la ciencia como lo comenta Rojas (2000):

-

La visión objetiva, que hacer ver las cosas y fenómenos en su propia realidad y no conforme al gusto o prejuicios del observador.

- $\quad$ El pensamiento lógico, que exige explicaciones de las causas de los fenómenos que sean razonables y verificables, excluyendo causas imposibles de comprobar. 
- El pensamiento critico, comparando las construcciones teóricas con los hechos observados.

La resolución de problemas puede generar también un potencial investigativo, si este se considera dentro de su formulación, una duda a la que hay que buscar evidencias para generar un respuesta y por ende el aprendizaje. Es el dicente el que debe indagar el procedimiento resolutivo y no el docente el que le da la solución, este nada más debe guiar el proceso. (Gil, et al., 1999)

Esta manera de aprendizaje de conceptos científicos, acerca al estudiante al proceso histórico y la situación problematizadora en la que se generó el conocimiento y al proceso de justificación del mismo. Sin embargo, bajo las estrategias tradicionales de enseñanza de las Ciencias, sólo se le da énfasis a la justificación de los fenómenos dejando de lado la contextualización (vinculación con la realidad cotidiana) del fenómeno. (Campanario, 2000). La investigación dirigida implica entonces todo un cambio de paradigma y de teorías de aprendizaje en el proceso de la enseñanza de las Ciencias, así como un cambio en el docente y en el estudiante de metodologías, conceptos y actitudes. (Pérez, 1993 y Gil, et al., 1999)

Sin dejar de lado, la necesidad de un cambio cultural, pues hasta el momento en torno a la enseñanza tradicional de las ciencias se ha creado toda una cultura, acrítica, mecanizada e impotente. La investigación dirigida rompe con los contradicciones epistemológicas y ontológicas bajo las cuales se desarrolla la enseñanza tradicional en su expresión general.

Campanario (2000) apoya la idea aquí expuesta, en la que las situaciones problematizadoras deben de generarse de las situaciones cotidianas, de manera que el y la estudiante logre pertinencia, arraigo y ganas de aprender algo nuevo que pueda explicar de manera satisfactoria ese fenómeno que tanto le intrigó.

Durante todo el proceso investigativo indudablemente surgirá en algún momento la necesidad de utilizar bibliografias para sustentar el proceso. El uso de tales recursos deben de ir acorde a la edad y a la etapa de desarrollo cognitivo en la que se encuentre el o la estudiante investigadora. (Campanario, 2000)

De nuevo, el docente adquiere un papel de mediador importante, debido a que estamos en una época en que la información está más cerca de las personas, más que en ninguna época de la historia de la 
humanidad. El o ella deben estar al lado de sus estudiantes y enseñarles a discriminar las buenas fuentes de información de aquellas cuyo contenido es incompleto o bien incorrecto.

El y la docente hoy más que nunca, deben generar espacios de crítica en el aula, en la que los y las estudiantes intercambien sus conocimientos y los contrasten (Gil, et al., 1999) de manera que así con ayuda del profesor o profesora puedan discernir el conocimiento más acertado por las teorías, de los conceptos erróneos.

Campanario (2000), así como Gil et al. (1999) aceptan la idea de enseñar los nuevos conceptos a partir de procesos investigativos. Lo definen como una nueva manera de abordar la resolución de problemas a través del proceso investigativo.

Campanario (2000) va más halla y propone que más que resolver un problema es enfrentársele y debe surgir de un ejercicio por parte del estudiante, de autocuestionamiento y de autoformulación de preguntas.

La investigación dirigida está basada en uno de los ejes centrales del hacer Ciencia: El motor del desarrollo de las teorias son los problemas (Furió y Guisasola, 2001). Como analogía, el motor del aprendizaje de un nuevo concepto son los problemas cotidianos.

Pérez (1993), Gil et al (1999) y Campanario y Moya (1999) establecen posibles secuencias lógicas para el proceso de mediación del conocimiento mediante la investigación dirigida, que a continuación se engloban:

1. Se plantea la situación problemática, el nuevo concepto o se puede plantear a través de una hipótesis (Furió y Guisasola, 2001),

2. Los y las estudiantes expresan sus ideas,

3. Se propone una estrategia (tratamiento científico) para abordar el nuevo aprendizaje: dentro de este paso se pueden plantear hipótesis, las estrategias (Que puede ser un diseño experimental) y se obtienen los nuevos resultados, luego se vuelven a contrastar los nuevos conceptos entre los y las investigadoras, lo que generará un muy rico conflicto cognitivo entre los conocimientos previos y los nuevos conocimientos,

4. Se plantean los nuevos conocimientos y se sintetizan y generan las respuestas, es importante relacionar los nuevos conceptos con los ya asimilados, expresar la utilidad de estos conceptos a otros fenómenos cotidianos, lo que hemos llamado "contextualizar" 


\section{Se plantean nuevos problemas.}

Como señala Gil (1994), el cambio conceptual adquiere ahora un carácter instrumental y deja de ser un objetivo en sí mismo: la investigación no se plantea para conseguir el cambio conceptual, sino para resolver un problema de interés, el cambio conceptual se produce a lo largo de todo el proceso como un resultado más, de ahí el énfasis en el necesario cambio metodológico que debe acompañar todo el proceso.

Sin embargo, se rechaza la idea de reducir todo al aprendizaje de un método científico como conjunto de reglas perfectamente definidas que se aplican mecánicamente (Gil, 1994). Ésta y otras formulaciones insisten también en el cambio actitudinal.

La Investigación Dirigida más que un método de enseñanza se puede considerar como un método de construcción del aprendizaje, el cuál brinda a los y las estudiantes las herramientas para el auto - aprendizaje, llegando a la premisa del aprender a aprender, generando autonomía y capacidad crítica en el discente investigador (Cañal y Porlan, 1987).

La Investigación Dirigida, es una metodología cobijada bajo un paradigma naturalista, fortalecido en la teoría del constructivismo, el aprendizaje de un concepto se construye de manera activa por parte del aprendiz y se hace basado en los conocimientos previos, lo que Pozo (1989) citado por Furió y Guisasola (2001) llama como un cambio conceptual.

La investigación se convierte entonces en una herramienta fundamental (más no única) para la construcción del conocimiento y el proceso de mediación pedagógica a partir de las concepciones previas. Se resalta así, la importancia que juega en el aprendizaje los conceptos previos de manera que el aprendizaje del nuevo conocimiento no se levanta de la nada, otra vez viéndose reflejada la teoría del constructivismo y del aprendizaje significativo, que aunque no se ha mencionado de manera de explicita en el texto, todo este hace alusión al aprendizaje de conceptos mediante la teoría del aprendizaje significativo de David Ausbel. (Cañal y Porlan, 1987 y Pérez, 1993)

Furió y Guisasola (2001) plantean que el aprendizaje de conceptos científicos se aleja entonces de la mera superficialidad (aprendizaje memorístico), cuando se aplica la metodología científica para aprender un concepto, consecuentemente lográndose la construcción del conocimiento. 
Pozo y Gómez (1998), Gil et al (1999) y Furió y Guisasola (2001), todos plantean el hecho de que el proceso de investigación Dirigida debe ser guiado por el docente y este se debe de realizar mediante grupos cooperativos formados por los y las estudiantes, donde estos adquieren entonces, el termino de "Investigadores Noveles", más que el de simples investigadores como era planteado anteriormente, su principal función es formar investigadores. Idea que no es muy descabellada, ya que para mejorar la realidad en la que se vive, una de las mejores herramientas es investigar qué es lo que está mal, proponer soluciones reales y aplicarlas, a la vez que se investiga como mejorar en lo que se es mejor. La Investigación Dirigida se convierte entonces en una estrategia metodológica que es aplicable a todos los aspectos de la vida cotidiana y por tanto en todas las áreas de la ciencia.

McComas (1998) plantea en Furió y Guisasola (2001), que el seguimiento del método científico como herramienta para la enseñanza de las Ciencias es un cambio epistemológico que se persigue para enseñar Ciencias como investigación; es la manera en que se trata de fundamentar la Investigación Dirigida, extraer estrategias procedimentales utilizadas por los científicos que permitan la construcción del conocimiento.

El profesor en todo el proceso investigativo, tiene una función muy importante debe presentar el problema y lo que puede ser más importante generar el interés (Ayuso y Banet, 2002) y generar lo que se podría llamar una lluvia de ideas antes de iniciar el proceso, luego basta con orientar el proceso investigativo. Este proceso puede entonces ser un muy buen medio de reconciliación, entre lo que Cañal y Porlan en 1987 denominaron un divorcio entre realidad próxima del alumno y la escuela. La ciencia en nuestro caso debe servir para explicar al alumno la realidad en la que vive, de modo que la Ciencia que se enseña en el aula tendrá un sentido para ellos generándose así el verdadero aprendizaje significativo.

Entonces la investigación dirigida también engloba métodos de enseñanza que Pozo y Gómez (1998), ya han definido, como es el caso del Aprendizaje por descubrimiento y el mismo conflicto cognitivo (al constatar muchas veces el o la estudiante que lo que el creía que ocurría en un fenómeno no es en realidad la explicación más verídica), evidenciándose así de manera certera la acción integralizadora que puede ser la aplicación de la investigación como estrategia en el proceso de construcción del conocimiento. 
En aquellas décadas del sesenta el Aprendizaje por Descubrimiento tenía por objetivo que los y las estudiantes disfrutaran el hacer ciencia. A pesar de que en estas épocas los avances de la psicología popularizaron la pedagogía de la acción o activismo, especialmente con aportes de Piaget, Bruner y Gagné (Pérez, 1993; Velásquez y López 2008; Leymonié 2009).

El gran fallo que tuvo esta estrategia de aprendizaje era pretender que el estudiante fuera un pequeño científico. Y algunas críticas hechas sobre este tipo de aprendizaje por descubrimiento son (Fernández 2002; Furió 2001):

- No tuvo en cuenta al profesor como director del proceso enseñanza - aprendizaje.

- Identifica al descubrimiento como consecuencia del aprendizaje inductivo

- Identifica al aprendizaje por descubrimiento como experiencia intuitiva.

- Prestó poca atención a los contenidos.

- Identifica al descubrimiento con aprendizaje mediante prácticas de laboratorio.

- Interpreta al descubrimiento como aprendizaje por ensayo - error.

No obstante, a las innumerables deficiencias presentadas, el aprendizaje por descubrimiento significó un despertar a nuevas propuestas de aprendizaje, constituyó el origen de la reestructuración, provocó una revisión crítica de la educación científica, intentó familiarizar a los estudiantes con el trabajo científico; aunque no tuvo en cuenta importantes aspectos del trabajo científico (Membiela 1997).

No obstante, la investigación Dirigida tiene la ventaja de que la duda o interrogante surge a partir del mismo estudiante, lo que genera mucho sentido de pertinencia y curiosidad por encontrar la respuesta, lográndose una verdadera construcción del conocimiento. Una de las herramientas para alcanzarlo puede ser el uso del laboratorio (aprendizaje por descubrimiento), donde el alumno o alumna disfrute verdaderamente del quehacer científico, sin pensar en los discentes como pequeños científicos.

Pérez (1993) establece que la investigación es una muy buena técnica, sin embargo advierte que el trabajo investigativo debe realizarse 
en grupos cooperativos de trabajo que sean pequeños, donde el profesor o profesora dirija, oriente y la final del proceso refuerce el conocimiento adquirido. El educador en todo el proceso puede alimentar la duda o el conflicto con un determinado concepto, aumentado así la curiosidad y las ganas por aprender por parte de los y as estudiantes.

La investigación dirigida puede ser un método usado por los docentes de ciencias para acercar a sus estudiantes a lo que es ser un científico, pero nunca pretender que lo sean en realidad, además es un muy buen método para acercar el conocimiento o conceptos científicos a la realidad cotidiana. (Campanario y Moya, 1999)

No se trata de crear la falsa imagen de que la Investigación Dirigida es la panacea para aprender y enseñar ciencias, es una estrategia que junto con otras estrategias, pueden generar en el o la estudiante el amor por las ciencias o bien, comprender lo vital que es saber ciencias para poder explicar todos los fenómenos naturales que ocurren en nuestro ambiente.

La Investigación Dirigida, es sólo una de las tantas estrategias que el docente puede usar, ya que en mucho depende del currículo oficial, del currículo oculto, planeamiento didáctico y objetivos que cada docente establezca en su práctica profesional. Y precisamente, aunque puede ser muy útil para enseñar algunos temas, puede que para otros no lo sea.

Esta estrategia también tiene sus dificultades entre estas destaca Campanario y Moya (1999): Implican esfuerzos que muchas veces los y las alumnas no están dispuestos a realizar, el tiempo para su aplicación es bastante amplio y otras veces la explicación del fenómeno puede no llamar la atención de los y las alumnas.

Además, otro de los problemas más importantes que suele plantear este enfoque es sin duda su alto nivel de exigencia al profesorado, lo que hace dificil su generalización. Pues, enseñar la ciencia como un proceso de investigación dirigida requiere una determinada concepción de la ciencia y de su enseñanza, que no suele estar muy extendida ni aceptada entre los docente.

La estrategia metodológica de Investigación Dirigida permite y fomenta la integración en el aula, es decir, procura la unión de grupo, bajo el concepto de investigación de "algo nuevo" aquello que para muchos es confuso, al final será claro y divertido para los pequeños investigadores. Además es posible pensar en la interdisciplinaridad de las diferentes áreas, más allá de las ciencias. 
Por ejemplo, investigar en ciencia implica el buscar en todo un contexto histórico y cultural, que le permite al investigador contar con la fundamentación científica necesaria para la construcción de su proceso de formación del conocimiento y la sistematización de su trabajo. Entonces, ¿Será capaz la Investigación Dirigida de integrar áreas como los estudios sociales, las artes, la matemática, la filosofia y demás áreas del saber en el proceso de construcción del conocimiento? O bien, ¿El conocimiento se construye de manera aislada?

Son cuestionamientos realmente interesantes, y por su puesto que, la Investigación Dirigida permite no sólo la integración de las diferentes áreas del saber, si no que consecuentemente interrelaciona estilos de aprendizaje e inteligencias múltiples es decir, unos estudiantes son mejores en matemática, otros son mejores en estudios sociales o en ciencias, de forma que, se construye y se comparte el conocimiento en grupo, fortaleciéndose la capacidad cognitiva de cada uno de los miembros del grupo que está haciendo investigación. (Acevedo 2006)

Por otra parte, el educador en su función de mediador, debe sin lugar a dudas formar parte de cada uno de los pequeños grupos de investigadores Nóveles, lo que implica, el bajar del lugar de quien "sabe todo" a el compañero que simplemente tiene un poco más de experiencia y puede guiar el proceso de manera satisfactoria.

\section{Conclusiones}

La Investigación Dirigida no es la estrategia perfecta mediante la cual, nosotros, los educadores de la Ciencia podemos sentirnos satisfechos, no es la barita mágica que soluciona todos nuestros problemas en el aula, simplemente es una de las muchas estratégicas metodológicas que busca romper con los paradigmas ambiguos de la enseñanza de la Ciencia, es una herramienta integradora, capaz de generar en nuestros y nuestras estudiante la habilidad y la pasión por la ciencia, en cualesquiera de sus especialidades.

Con la Investigación Dirigida comienza a ser posible avanzar en la solución de los problemas que plantea la enseñanza de las Ciencias ya que dicha enseñanza se contempla como situación problemática que exige investigación, es decir, el cuestionamiento de las evidencias. (Gil et al, 1999) 
La aplicación de la Investigación Dirigida radica en que los estudiantes puedan explicar fenómenos de su realidad inmediata, lo cuál es muy importante para reafirmar mediante una aplicación objetiva los conceptos vistos en la clase, concientizando sobre la aplicabilidad de la ciencia en nuestras vidas.

Los diversos enfoques alternativos a la enseñanza tradicional insisten en la necesidad de que los alumnos desempeñen un papel más activo en clase; y este método de enseñanza por Investigación dirigida puede consistir en tareas diversas, desde realizar experiencias hasta resolver problemas, y se concibe como una elaboración o aplicación de los conocimientos que constituya una alternativa a la memorización simple de los mismos (Smith, 1993).

Por otra parte, parece existir consenso en que esta propuesta requiere, en general, más tiempo para desarrollar los contenidos que el que se requiere en la enseñanza tradicional. Pero la aplicación de este método dependerá de la creatividad de cada docente dentro su contexto de aula; y por supuesto de la teoría pedagógica en la cual cada educador se apoye pues, como se ha comentado este método tiene su fundamentación en teoría Constructivista que no todos los docente aceptan.

Como conclusión general se puede decir que la tendencia actual en la Enseñanza de las Ciencias es orientar el aprendizaje como construcción de conocimientos científicos en la que asocia el aprendizaje al tratamiento de situaciones problemáticas, sea cuál sea la estrategia de enseñanza que se utilice. Además tales conocimientos científicos deben de estar aplicados a la realidad cotidiana para que estos tengan algún significado para el estudiante. (Gil, Furió, et al, 1999; Campanario, 2000).

"El aprendizaje de las ciencias es concebido asi no como un simple cambio conceptual, sino como un cambio a la vez conceptual, metodológico y actitudinal. Una integración de la teoría la practica y los problemas, en un proceso único de construcción de conceptos cientificos." (Gil, et al, 1999, p. 317) 


\section{REFERENCIAS}

Acevedo, J. 2006. Investigación científica, naturaleza de la ciencia y enseñanza de las ciencias, Rev. Eureka. Enseñ. Divul. Cien., 3(2): 306-311

Alfono, C. 2004. Familiarización de los estudiantes con la actividad cientifico-investigadora: Método dinámico para caracterizar el movimiento de traslación de un cuerpo. Rev. Enseñanza de las Ciencias, 3 (1): 1-13

Ayuso, G. y E., Banet, 2002, Alternativas a la enseñanza de la genética en educación secundaria, Enseñanza de las Ciencias, 20 (1): 133 - 157.

Campanario, J., 1999, El desarrollo de la metacognición en el aprendizaje de las ciencias: Estrategias para el docente y actividades orientadas para el alumno, Enseñanza de las Ciencias, 18 (3): $369-380$.

Campanario, J. y A., Moya, 1999, ¿Cómo enseñar ciencias? Principales tendencias y propuestas, Enseñanza de las Ciencias, 17 (2): 179 - 192.

Cañal, P. y R., Porlan, 1987, Investigando la realidad proxima: un modelo didáctico alternativo, Enseñanza de las Ciencias, 5 (2): 89 - 96.

Diego-Rasilla, F. 2004. El método cientifico como recurso pedagógico en el bachillerato: haciendo ciencia en la clase de biologia. Rev. Pulso 27: 111-118.

Driver, R, 1986, Psicología Cognoscitiva y Esquemas Conceptuales de los Alumnos, Enseñanza de las Ciencias, 4 (1), 3 - 15.

Fernández, J. 2002. Visiones deformadas de la ciencia transmitidas por la enseñanza. Rev. Enseñanza de las ciencias. Vol.2 (2): 477-488.

Furió, C. 2001. Finalidad de la enseñanza de las ciencias en la secundaria obligatoria: ¿Alfabetización científica o preparación propedéutica? Rev. Enseñanza de las ciencias Vol.2 (2): 365-376.

Furió, C. y Guisasola, J., 2001, La enseñanza del concepto de campo eléctrico basada en un modelo de aprendizaje como investigación orientada. Enseñanza de las Ciencias, 19 (2): $319-334$.

Gil, D. 1994. Relaciones entre conocimiento escolar y conocimiento cientifico. Investigación en la Escuela 23: 17-32.

Gil, D., C, Furió., P, Valdéz., J, Martines - Torregrosa., J, Guisasola., E, Gonzáles., A, Dumas - Carre., M, Goffard., A, Pessoa., 1999, ¿Tiene sentido seguir distinguiendo entre aprendizaje de conceptos, resolución de problemas de lápiz y papel y realización de prácticas de laboratorio?, Enseñanza de las Ciencias, 17 (2): $311-320$.

Hewson, P.1987. Conceptions of teaching: Implications for teacher's educations. Rev. International Journal of Science education $175-184$.

Leymonié, J. 2009. Aportes para la enseñanza de las ciencias. ( ${ }^{a}$. ed.), Editorial LLECE. Santiago, Chile. Trabajo realizado para la UNESCO.

Membiela, P. 1997. Una revisión del movimiento educativo ciencia-tecnología-sociedad. Rev. Enseñanza de las ciencias 15 (1): 51-57.

Moreira, M., Greca, I., 2003, Cambio Conceptual: Análisis Critico y Propuestas a la Luz de Teoría del Aprendizaje Significativo, Ciencia y Educaçao, 9 (2), 301 315 . 
Pérez, G., 1993, Contribución de la historia y filosofia de las ciencias al desarrollo de un modelo enseñanza / aprendizaje como investigación. Enseñanza de las Ciencias, 11 (2): $197-212$.

Posner, G., Strike, P., Hewson, P., Gertzog, W., 1982, Accommodation of a Scientific Conception: Toward a Theory of Conceptual Change, Science Education, 66 (2): $211-227$.

Pozo, I., Carretero, M., 1987, Del Pensamiento Formal a las Concepciones Espontáneas ¿Qué Cambia en la Enseñanza de la Ciencia? (Versión electrónica), Infancia y Aprendizaje, 38, 35 - 52.

Pozo, I., Gómez, J., 1998, Aprender a Enseñar Ciencias (1ª. ed.), Ediciones Morata, Madrid, España.

Rojas, M. 2000. La ciencia y la sociedad mexicana. Rev. Ciencia UANL 4:127-129.

Smith, E. 1993. Estrategias de enseñanza asociadas con el cambio conceptual en el aprendizaje en ciencias. Journal of Research in Science Teaching 30: 11-126.

Velásquez, A. y E. López. 2008. Principales tenencias de la Educación Cientifica en las últimas cuatro décadas, Rev. Investigación e Innovación en la Enseñanza de las Ciencias. Vol. 2 (1): 82-86. 